\title{
Nutrition status in adult Chilean population: economic, ethnic and sex inequalities in a post-transitional country
}

\author{
María F Mujica-Coopman ${ }^{1}$, Deborah Navarro-Rosenblatt ${ }^{2}$, Sandra López-Arana ${ }^{3}$ ๑ and \\ Camila Corvalán ${ }^{4, *}$ \\ 'Food, Nutrition and Health, Faculty of Land and Food Systems, The University of British Columbia, 2205 East Mall, \\ Vancouver, V6T 1 Z4 BC, Canada: ${ }^{2}$ School of Public Health, University of Chile, Avenida Independencia 1027, \\ Independencia, Santiago, Chile: ${ }^{3}$ Department of Nutrition, Faculty of Medicine, University of Chile, Avenida \\ Independencia 1027, Independencia, Santiago, Chile: ${ }^{4}$ Institute of Nutrition and Food Technology (INTA), \\ University of Chile, El Líbano 5524, Santiago, Chile
}

Submitted 7 January 2019: Final revision received 13 September 2019: Accepted 16 October 2019: First published online 5 March 2020

\begin{abstract}
Objective: To assess the relationship between malnutrition, socioeconomic status (SES) and ethnicity in Chilean adult population.

Design: Nationally representative survey (ENS) conducted in 2016-2017. Sociodemographic information, weight, height and hemoglobin ( $\mathrm{Hb}$ ) were measured (2003 ENS). Excess weight was defined as BMI $\geq 25 \mathrm{~kg} / \mathrm{m}^{2}$. Undernutrition included underweight (BMI $<18.5 \mathrm{~kg} / \mathrm{m}^{2}$ ), short stature (height $<1.49 \mathrm{~m}$ in women and $<1.62 \mathrm{~m}$ in men) or anaemia $(\mathrm{Hb}<12 \mathrm{~g} / \mathrm{l})$. Education and household income level were used as indicators of SES; ethnicity was self-reported. We applied linear combinations of estimators to compare the prevalence of excess weight and undernutrition by SES and ethnicity.

Setting: Chile.

Participants: In total, 5082 adults $\geq 20$ years (64\% women) and 1739 women $\geq 20$ years for anaemia analyses.

Results: Overall, $>75 \%$ of women and men had excess weight. Low SES women either by income or education had higher excess weight $((82 \cdot 0(77 \cdot 1,86 \cdot 1) v .65 \cdot 0$ $(54 \cdot 8,74 \cdot 1))$ by income; $(85.3(80 \cdot 6,89 \cdot 0) v .68 \cdot 2(61 \cdot 6,74 \cdot 1) \%)$ by education) and short stature (20-49years; $31(17 \cdot 9,48 \cdot 2)$ v. $5 \cdot 2(2 \cdot 2,11.4)$ by education); obesity was also more frequent among indigenous women (20-49years; $55 \cdot 8(44.4,66 \cdot 6) v \cdot 37 \cdot 2(32 \cdot 7,42 \cdot 0) \%)$ than non-indigenous women. In men, excess weight did not significantly differ by SES or ethnicity, but short stature concentrated in low SES (20-49 years; $47 \cdot 6(24 \cdot 6,71 \cdot 6) v .4 \cdot 5(2 \cdot 1,9 \cdot 5)$ by education) and indigenous men $(21.5(11.9,5 \cdot 5,11.9) v .8 \cdot 2(5.5,11.9))(P<0.05$ for all $)$.

Conclusions: In Chile, malnutrition is disproportionately concentrated among women of low SES and indigenous origin; these inequalities should be considered when implementing prevention policies.
\end{abstract}

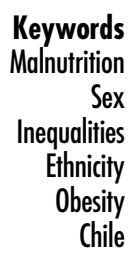

In the 1960 s, Chilean population was affected by a high incidence of infectious diseases (300 per 100.000 inhabitants) and prevalence of infant mortality of $24 \%{ }^{(1)}$. Additionally, $37 \%$ of children had undernutrition ${ }^{(2)}$ and approximately $20 \%$ were anaemic $^{(3)}$. Public health policies and programs were focused on decreasing maternal-child mortality, undernutrition and micronutrient deficiency ${ }^{(4)}$.
However, during the last decades, Chile has faced an extremely rapid nutrition transition ${ }^{(4,5)}$.

Currently, Chile leads in obesity burden, with one of the highest prevalence worldwide ${ }^{(6)}$. According to the latest National Health Survey, 31\% of adult population ( $\geq 15$ years) is obese, whereas the prevalence of overweight is almost $40 \%{ }^{(7)}$. The prevalence of excess weight,

Disclaimer: This paper was published as part of a Supplement in the Journal of Public Health Nutrition, publication of which was supported partially by SLAN CAPÍTULO MEXICO A.C. The papers included in this supplement were invited by the Guest Editor and have undergone the standard journal formal review process. They may be cited. 
defined as the combined prevalence of overweight and obesity, has increased from $6 \%$ in the $1980 \mathrm{~s}^{(5)}$ to $71 \%$ in $2016^{(7)}$. Additionally, $9 \%$ of children $<5$ years are overweight, increasing this prevalence to $31 \%$ in children $>5$ years ${ }^{(8,9)}$. In contrast, the prevalence of anaemia is no longer a public health issue in Chilean women $(<5 \%)$ and neither is stunting or underweight ${ }^{(10,11)}$. Thus, Chile is considered as a country facing post-transitional stages in the nutrition transition ${ }^{(12)}$. This nutrition situation contrast with the reality of several Latin-American neighbouring countries that are facing early stages of this transition, where undernutrition and obesity coexist at the individual, household and country levels ${ }^{(13)}$. In Ecuador, 9\% of the adult population have excess weight and anaemia at the individual or household level ${ }^{(14)}$, while in Guatemala, $48 \%$ of children $<5$ years are stunted, $49 \%$ have anaemia and $5 \%$ have excess weight ${ }^{(15)}$, whereas in Uruguay, $10 \%$ of children are stunted and have excess weight ${ }^{(16)}$.

This period of nutritional change in Chile has been concomitant with rapid economic growth characterised by the reduction in poverty rates (i.e. extreme poverty defined as a monthly per-capita income of \$US 154 or less decreased from $3.5 \%$ in 2006 to $2.6 \%$ in 2015$)^{(17,18)}$. However, according to the 2015 OCDE Inequality Report ${ }^{(19)}$, Chile has the highest inequality level in the region, showing the lowest reduction in the GINI coefficient ( $-3 \cdot 1 \%$ ), a well-known inequality index, in the period 2002-2011, compared with Argentina (-10.3\%), Bolivia (-12.4\%), Ecuador (-3.8\%) and Uruguay $(-5 \cdot 4 \%)^{(20)}$.

The relationship between socioeconomic status (SES) and several nutritional status indicators such as excess weight has been previously described ${ }^{(21-24)}$. In Chile, a study including more than 1500000 Chilean children (mean age $76 \cdot 3$ (SD 4.35) months) showed that poorest children had a higher prevalence of stunting compared with wealthier children $^{(25)}$, whereas Azar et al. ${ }^{(26)}$ found that high-middle SES adolescents assessed by the mother's education level were less likely to be overweight or obese, compared with those with a low socioeconomic level. Similar results were found in a cohort study which included adults living in the Valparaíso Region of Chile, where low SES women showed a significant increase in BMI and waist circumference (WC) compared with high SES women in a 10-year period; in men, neither BMI nor WC differs by $\mathrm{SES}^{(27)}$.

In addition to the influence of SES, previous studies have reported that ethnicity may play a key role on nutritional status of Chilean population. Currently, $10 \%$ of Chilean population identifies themselves as indigenous, being the largest preference Mapuche ${ }^{(28)}$. Bustos et al. ${ }^{(25)}$ reported that first-grade school Mapuche children were shorter than non-Mapuche children. Additionally, a recent longitudinal study in which pre-schooler children was followed for 2 years indicated that being indigenous was a risk factor for developing overweight or obesity at 6 years ${ }^{(29)}$. Similar findings were reported at school-age children where indigenous children had a $6 \%$ higher risk of being obese compared with non-indigenous children ${ }^{(25)}$. Nonetheless, there is still controversy on whether part of the observed nutritional differences by ethnicity are actually due to SES differences ${ }^{(30)}$.

To date, the relationship between malnutrition, SES and ethnicity has not been studied in a Chilean national representative sample. Therefore, the aim of the current study is to assess the relationship between all malnutrition forms (e.g. excess weight, anaemia), socioeconomic indicators defined by the income and education level and ethnicity in a representative sample of Chilean women and men. Our goal is to describe how different forms of malnutrition are present on a post-transitional country that has alarming SES, ethnicity and sex inequalities.

\section{Population and methods}

\section{Double burden series}

- The current article corresponds to one of a series of papers exploring the double burden of malnutrition in LAC; therefore, sample, methods and data analyses definitions were made on agreement with all other countries. Some of these definitions or categories do not apply to the context of Chile but have been kept in the manuscript to keep comparability across articles.

\section{Data source and sampling}

\section{National Health Survey 2016-2017: nutritional status} and demographic characteristics

Data on height, weight, education, monthly household income level and ethnicity were obtained for adults ( $>20$ years) from the latest available Chilean National Health Survey (ENS) conducted in 2016-2017 ${ }^{(7)}$.

The 2017 ENS included 6233 participants aged $>15$ years, using a random household sampling (multistage, cluster sample and stratification by urban-rural and region population), had a high rate of response of $85 \%^{(10)}$. For the current study, subjects were excluded according to the following criteria: age $<20$ years ( $n 355)$, no available anthropometric measurements $(n 750)$ and no available education level ( $n$ 46). Final sample included 5082 adults, 3257 women and 1825 men.

Nutritional status. Subjects were classified as underweight, overweight and obese using BMI $\left(\mathrm{kg} / \mathrm{m}^{2}\right)$ following the WHO criteria ${ }^{(31)}$. Excess weight was defined as BMI $\geq 25 \mathrm{~kg} / \mathrm{m}^{2}$. Given the physiological changes in height during late adulthood ${ }^{(32)}$ and also the relationship between the obstetric risk and short stature ${ }^{(33)}$, we only estimated the short stature prevalence in women and men between 20 and 49 years. Short stature was classified according to the tenth percentile of the sample distribution (height $<1.49 \mathrm{~m}$ for women and $<1.62 \mathrm{~m}$ for men). Undernutrition was defined as the presence of either underweight, short stature or anaemia (see below for definition). 
Socioeconomic status. Education and monthly household income levels were considered as indicators of SES. Education level of the participants was classified as: (i) low ( $<8$ years); (ii) medium ( $8-12$ years) and (iii) high ( $>13$ years) based on self-reported years of completed schooling. All participants were asked to report their monthly household income using eleven categories that ranged from less than \$US 77.999 Chilean Pesos (CLP) (about \$US 116) to more than \$US 1.573.000 CLP (about $\$$ US 2348). We categorised the monthly household income in four levels as low (less than \$US 295.999 CLP), medium (between \$US 296.000 and \$US 1.000.000 CLP) high (more than \$US 1.000.000 CLP) and non-responders that accounted for a $14.6 \%$ of final sample. Additionally, we provided descriptive information about bare-earth floor, use of firewood or carbon for cooking, public sanitary sewer system, public water network connection and electricity as an indirect indicator of SES.

Ethnicity. Ethnicity was classified by asking 'Do you belong or are you a descendant of any of the nine indigenous peoples recognizes by law in Chile?' which had ten possible answers (i.e. Aymara, Rapa-Nui (Pascuenses), Quechua, Mapuche, Acatameno (Lincai Antai), Coya, Kawesqar (Alacalufes), Yagan (Aymara) or Diaguita). We dichotomised the variable into 'yes' (belong to any of the nine indigenous peoples) and 'no' (do not belong).

\section{National Health Survey 2003: anaemia information}

Due to the lack of hemoglobin (Hb) information in 20162017 ENS, anaemia and the corresponding education information was obtained from the last national survey that had micronutrient status information that was the $2003 \mathrm{ENS}^{(10)}$. The 2003 ENS is a cross-sectional national representative sample which included 3619 participants (women, $n$ 1973), aged $\geq 17$ years, stratified by urban-rural and region population and had a rate of response of $90 \%$. Sampling procedures followed a multistage, stratified and cluster design.

Anaemia. Given the vulnerability of developing iron deficiency and anaemia in women and the median age of menopause in Chilean women ${ }^{(34)}$, Hb concentration was determined only in women population $\geq 20$ years. Women were excluded if they were $<20$ years ( $n 86)$, had no available $\mathrm{Hb}$ measurement $(n 144)$ or education information ( $n$ 4). In total, we analysed available $\mathrm{Hb}$ data of 829 (2049 years) and 910 (>49 years) women. Hb concentration was determined by colorimetric method ${ }^{(10)}$. Anaemia was defined according to the $\mathrm{WHO}$ criteria as $\mathrm{Hb}$ concentration $<12 \mathrm{~g} / \mathrm{dL}^{(35)}$. Hb values were not adjusted by altitude, because this information was not available in the 2003 ENS. Women were classified with a low ( $<8$ years), medium ( $8-12$ years) and high ( $>13$ years) education level based on self-reported years of completed schooling.

\section{Statistical analyses}

We estimated the prevalence and 95\% CIs of indicators of overweight, obesity, excess weight, underweight, short stature and anaemia according to sex, ethnicity, age groups, monthly household income and education levels. Continuous and categorical variables were described as means or percentages and $95 \% \mathrm{CI}$, respectively. To compare our findings with the results of other Latin American countries included in this 'Double Burden Series', we classified adult population by age categories: $20-49$ years and $>49$ years. Differences in means and proportions of excess and undernutrition by SES and ethnicity were tested using the test for linear combinations ('lincom' command in Stata 12.0). The statistical significance level was set at $P<0.05$. All the statistical analyses were performed with Stata version 12 (Stata Corp.) incorporating appropriate survey sample weight.

\section{Results}

Age, education level, ethnicity and household characteristics of the sample stratified by income levels are shown in Table 1. The average age of 20-49year samples was 34.6 years in women and 33.2 years in men, while in adults $>49$ years it was 62.9 years in women and 63.1 years in men. Additionally, $8 \%$ of the subjects identified themselves as indigenous. Most of the participants had medium education level (women (53.1\%) and men (44.7\%)) and were classified as medium monthly household income level. Younger adults (20-49 years) had higher educational levels compared with older adults ( $>49$ years). As expected, there was a positive relationship between education level and monthly household income levels and household characteristics; for example, women and men with a highest income had highest education, and women and men with lower income were more likely to use firewood or carbon for cooking and had less water and sanitation facilities than women and men that had a highest income level. Interestingly, $12 \cdot 4 \%$ of low SES adults identified themselves as indigenous, whereas only $3 \%$ of high SES adults indicated being indigenous.

\section{Nutrition status in adult Chilean population}

Most of the population $(78.7 \%$ in women and $77.2 \%$ in men) had excess weight, whereas short stature and anaemia were below 13 and $8 \%$, respectively. Underweight was almost negligible ( $<2 \%$ in both sexes) (Table 2$)$. Obesity was more prevalent in women than in men, while the opposite was seen for overweight prevalence $(P<0.05)$. Furthermore, in both sexes, the prevalence of obesity increased with age.

\section{Nutrition status, socioeconomic status and ethnicity in women}

We observed an inverse association between SES (either by income or educational level) with excess weight $(<49$ years) and obesity ( $>20$ years) $(P<0.05)$ (Table 2 ; Figs 1 and 2). Obesity was also more frequent among indigenous than non-indigenous young women ( $<49$ years; 
Table 1 Sample characteristics by household income in Chile based on the 2016-2017 National Health Survey

\begin{tabular}{|c|c|c|c|c|c|c|c|c|c|c|}
\hline & & & \multicolumn{8}{|c|}{ Income level } \\
\hline & \multicolumn{2}{|c|}{ All } & \multicolumn{2}{|c|}{ Low } & \multicolumn{2}{|c|}{ Medium } & \multicolumn{2}{|c|}{ High } & \multicolumn{2}{|c|}{ Without information } \\
\hline & $\begin{array}{l}\text { Average age } \\
\text { or } \%\end{array}$ & $95 \% \mathrm{Cl}$ & $\begin{array}{l}\text { Average age } \\
\text { or } \%\end{array}$ & $95 \% \mathrm{Cl}$ & $\begin{array}{c}\text { Average age } \\
\text { or } \%\end{array}$ & $95 \% \mathrm{Cl}$ & $\begin{array}{l}\text { Average age } \\
\text { or } \%\end{array}$ & $95 \% \mathrm{Cl}$ & $\begin{array}{l}\text { Average age } \\
\text { or } \%\end{array}$ & $95 \% \mathrm{Cl}$ \\
\hline Women $20-49$ years $(n 1489)$ & & & \multicolumn{2}{|c|}{$n 337$} & \multicolumn{2}{|c|}{$n 681$} & \multicolumn{2}{|c|}{$n 235$} & \multicolumn{2}{|c|}{$n 237$} \\
\hline $\begin{array}{l}\text { Age (years) } \\
\text { Education level }\end{array}$ & $34 \cdot 6$ & $33 \cdot 9,35 \cdot 4$ & $35 \cdot 9$ & $34 \cdot 5,37 \cdot 3$ & $35 \cdot 1$ & $34 \cdot 0,36 \cdot 2$ & 33.3 & $31 \cdot 6,35 \cdot 1$ & $32 \cdot 6$ & $30.5,34.7$ \\
\hline Low ( $<8$ years) & 7.4 & $5 \cdot 5,10 \cdot 2$ & $15 \cdot 5$ & $10 \cdot 3,22 \cdot 7$ & $7 \cdot 6$ & $4 \cdot 6,12 \cdot 4$ & 0.0 & $0.0,0.0$ & 3.2 & $1 \cdot 3,7.4$ \\
\hline Medium (8-12 years) & 55.9 & $51 \cdot 2,60 \cdot 4$ & 66.9 & $57 \cdot 6,75 \cdot 0$ & 62.9 & $56 \cdot 1,69 \cdot 2$ & $37 \cdot 8$ & $26 \cdot 8,50 \cdot 1$ & 37.8 & $26 \cdot 9,50 \cdot 1$ \\
\hline High (13 years or more) & $36 \cdot 7$ & $32 \cdot 3,41 \cdot 3$ & $17 \cdot 6$ & $11 \cdot 1,26 \cdot 7$ & 29.5 & $23 \cdot 7,36 \cdot 1$ & 62.2 & $49 \cdot 8,73 \cdot 1$ & 59.0 & $47 \cdot 0,70 \cdot 3$ \\
\hline Women $>49$ years $(n 1768)$ & & & \multicolumn{2}{|c|}{$n 690$} & \multicolumn{2}{|c|}{$n 672$} & \multicolumn{2}{|c|}{$n 104$} & \multicolumn{2}{|c|}{$n 302$} \\
\hline $\begin{array}{l}\text { Age (years) } \\
\text { Education level }\end{array}$ & $62 \cdot 9$ & $62 \cdot 1,63 \cdot 7$ & $64 \cdot 0$ & $62 \cdot 8,65 \cdot 2$ & 61.5 & $60 \cdot 1,62 \cdot 9$ & 63.8 & $60 \cdot 7,66 \cdot 9$ & $63 \cdot 1$ & $60 \cdot 9,65 \cdot 3$ \\
\hline Low (<8 years) & 38.7 & $34 \cdot 7,42 \cdot 9$ & $54 \cdot 1$ & $47 \cdot 9,60 \cdot 1$ & $28 \cdot 4$ & $22 \cdot 2,35 \cdot 5$ & $10 \cdot 9$ & $5 \cdot 0,22 \cdot 1$ & $36 \cdot 0$ & $26 \cdot 2,47 \cdot 2$ \\
\hline Medium ( $8-12$ years) & $49 \cdot 4$ & $45 \cdot 0,53 \cdot 9$ & $42 \cdot 3$ & $36 \cdot 4,48 \cdot 5$ & 58.4 & $50 \cdot 6,65 \cdot 7$ & $42 \cdot 9$ & $26 \cdot 7,60 \cdot 9$ & $48 \cdot 1$ & $36 \cdot 5,60 \cdot 0$ \\
\hline High ( 13 years or more) & 11.9 & $9 \cdot 3,15 \cdot 1$ & 3.6 & $2 \cdot 0,6 \cdot 5$ & 13.2 & $8.9,19 \cdot 2$ & $46 \cdot 2$ & $29 \cdot 9,63 \cdot 2$ & $15 \cdot 9$ & $8 \cdot 9,26.6$ \\
\hline Men $20-49$ years $(n 842)$ & & & \multicolumn{2}{|c|}{$n 128$} & \multicolumn{2}{|c|}{$n 357$} & \multicolumn{2}{|c|}{$n 226$} & \multicolumn{2}{|c|}{$n 131$} \\
\hline $\begin{array}{l}\text { Age (years) } \\
\text { Education level }\end{array}$ & 33.2 & $32 \cdot 4,34 \cdot 1$ & $36 \cdot 1$ & $34 \cdot 3,37 \cdot 8$ & 33.0 & $31 \cdot 7,34 \cdot 4$ & $32 \cdot 7$ & $31 \cdot 2,34 \cdot 1$ & $32 \cdot 1$ & $29 \cdot 6,34.6$ \\
\hline Low (<8 years) & 4.9 & $3 \cdot 0,8 \cdot 0$ & $18 \cdot 5$ & $10 \cdot 4,30 \cdot 8$ & $3 \cdot 2$ & $1 \cdot 3,7 \cdot 3$ & 2.5 & $0.0,15 \cdot 3$ & 0.0 & $0.0,0.0$ \\
\hline Medium ( $8-12$ years) & $52 \cdot 4$ & $47 \cdot 0,57 \cdot 8$ & 63.0 & $50 \cdot 7,73 \cdot 8$ & $62 \cdot 1$ & $53 \cdot 8,69 \cdot 7$ & 36.9 & $26 \cdot 5,48 \cdot 7$ & $42 \cdot 6$ & $29 \cdot 9,56 \cdot 4$ \\
\hline High ( 13 years or more) & $42 \cdot 7$ & $37 \cdot 3,48 \cdot 2$ & 18.5 & $11 \cdot 3,28 \cdot 8$ & 34.7 & $27 \cdot 3,43 \cdot 0$ & $60 \cdot 6$ & $48 \cdot 6,71 \cdot 4$ & $57 \cdot 1$ & $43.3,69.8$ \\
\hline Men $>49$ years $(n 787)$ & & & \multicolumn{2}{|c|}{$n 236$} & \multicolumn{2}{|c|}{$n 475$} & \multicolumn{2}{|c|}{$n 137$} & \multicolumn{2}{|c|}{$n 135$} \\
\hline $\begin{array}{l}\text { Age (years) } \\
\text { Education level }\end{array}$ & 63.1 & $62 \cdot 1,64 \cdot 2$ & $66 \cdot 5$ & $64 \cdot 6,68 \cdot 3$ & $62 \cdot 6$ & $60 \cdot 8,64 \cdot 3$ & $59 \cdot 4$ & $57 \cdot 9,60 \cdot 9$ & 62.9 & $60 \cdot 4,65.5$ \\
\hline Low (<8 year) & $30 \cdot 8$ & $26 \cdot 1,35 \cdot 9$ & $62 \cdot 2$ & $53.8,69.9$ & $26 \cdot 8$ & $19 \cdot 7,35 \cdot 3$ & 0.3 & $0.0,1 \cdot 3$ & $20 \cdot 8$ & $12 \cdot 8,31 \cdot 9$ \\
\hline Medium (8-12 years) & 47.9 & $42 \cdot 3,53 \cdot 5$ & $36 \cdot 6$ & $28 \cdot 9,45 \cdot 0$ & $60 \cdot 0$ & $51 \cdot 3,68 \cdot 1$ & 38.1 & $21 \cdot 9,57 \cdot 5$ & $35 \cdot 0$ & $23 \cdot 9,48 \cdot 1$ \\
\hline High ( 13 years or more) & $21 \cdot 3$ & $16 \cdot 6,27 \cdot 0$ & 1.3 & $0.0,3 \cdot 2$ & $13 \cdot 2$ & $8 \cdot 3,20 \cdot 4$ & 61.6 & $42 \cdot 3,77 \cdot 8$ & $44 \cdot 2$ & $29 \cdot 9,59 \cdot 5$ \\
\hline \multicolumn{11}{|l|}{ Households ( $n$ 4886) } \\
\hline \multicolumn{11}{|l|}{ Ethnicity } \\
\hline Indigenous & 7.6 & $6 \cdot 6,8 \cdot 8$ & $12 \cdot 4$ & $10 \cdot 1,15 \cdot 2$ & 7.6 & $6 \cdot 0,9 \cdot 6$ & 3.0 & $1 \cdot 6,5 \cdot 4$ & $5 \cdot 3$ & $3 \cdot 3,8 \cdot 2$ \\
\hline Non-indigenous & $92 \cdot 4$ & $91 \cdot 2,93 \cdot 4$ & 87.6 & $84.8,89 \cdot 9$ & 92.4 & $90 \cdot 4,94.0$ & $97 \cdot 0$ & $94 \cdot 6,98 \cdot 4$ & 94.7 & $91 \cdot 8,96 \cdot 7$ \\
\hline \multicolumn{11}{|l|}{ Household characteristics } \\
\hline Bare-earth floor & 0.05 & $0.0,0.1$ & 0.2 & $0.0,0.5$ & 0.0 & $0.0,0.0$ & 0.0 & $0.0,0.0$ & 0.08 & $0.0,0.2$ \\
\hline $\begin{array}{l}\text { Use of firewood or carbon for } \\
\text { cooking }\end{array}$ & $5 \cdot 1$ & $4 \cdot 3,6 \cdot 0$ & 13.0 & $10 \cdot 7,15 \cdot 8$ & 3.0 & $2 \cdot 3,4 \cdot 0$ & $1 \cdot 1$ & $0.4,3.0$ & $2 \cdot 7$ & $1 \cdot 3,5 \cdot 6$ \\
\hline Public sanitary sewer system & $89 \cdot 4$ & $88 \cdot 0,90 \cdot 6$ & $79 \cdot 4$ & $76 \cdot 0,82 \cdot 5$ & 90.5 & $88 \cdot 3,92 \cdot 4$ & $97 \cdot 4$ & $95 \cdot 4,98 \cdot 5$ & 93.5 & $90 \cdot 4,95 \cdot 6$ \\
\hline $\begin{array}{l}\text { Public water network connected } \\
\text { to household }\end{array}$ & 93.8 & $92 \cdot 6,94 \cdot 8$ & $86 \cdot 8$ & $84 \cdot 0,89 \cdot 2$ & $94 \cdot 3$ & $92 \cdot 2,96 \cdot 0$ & $99 \cdot 3$ & $98 \cdot 5,99 \cdot 7$ & $97 \cdot 3$ & $95 \cdot 3,98.5$ \\
\hline Electricity & 94.9 & $93 \cdot 7,95 \cdot 9$ & $91 \cdot 3$ & $88 \cdot 6,93 \cdot 4$ & 94.8 & $92 \cdot 6,96 \cdot 4$ & 98.3 & $94.8,99.4$ & 97.4 & $94.9,98.0$ \\
\hline
\end{tabular}




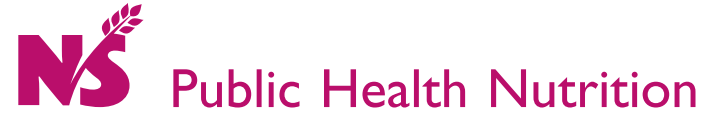

Table 2 Malnutrition's prevalence by household income, education level and ethnicity among adult women and men in Chile based on the 2016-2017 National Health Survey

\begin{tabular}{|c|c|c|c|c|c|c|c|c|c|c|c|c|c|c|}
\hline & & & \multicolumn{6}{|c|}{ Excess of weight } & \multicolumn{6}{|c|}{ Undernutrition } \\
\hline & \multicolumn{2}{|c|}{ All } & \multicolumn{2}{|c|}{ Overweightll } & \multicolumn{2}{|c|}{ Obesityף } & \multicolumn{2}{|c|}{ Excess weight†† } & \multicolumn{2}{|c|}{ 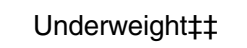 } & \multicolumn{2}{|c|}{ Short stature§§ } & \multicolumn{2}{|c|}{ AnaemiallII } \\
\hline & $n$ & $\% \S$ & $\%$ & $95 \% \mathrm{Cl}$ & $\%$ & $95 \% \mathrm{Cl}$ & $\%$ & $95 \% \mathrm{Cl}$ & $\%$ & $95 \% \mathrm{Cl}$ & $\%$ & $95 \% \mathrm{Cl}$ & $\%$ & $95 \% \mathrm{Cl}$ \\
\hline \multicolumn{15}{|l|}{ Women $20-49$ years } \\
\hline $\begin{array}{l}\text { All } \\
\text { Household income }\end{array}$ & 1489 & $100 \cdot 0$ & $36 \cdot 7$ & $32 \cdot 3,41 \cdot 3$ & $38 \cdot 8$ & $34.5,43.3$ & $75 \cdot 5$ & $71 \cdot 4,79 \cdot 2$ & $1 \cdot 2$ & $0.5,3.0$ & $12 \cdot 4$ & $9 \cdot 3,16 \cdot 3$ & $7 \cdot 2$ & $0 \cdot 0,16 \cdot 1$ \\
\hline Low & 337 & $22 \cdot 6$ & 40.9 & $32 \cdot 3,50 \cdot 1$ & $41.8 \dagger$ & $33 \cdot 4,50 \cdot 7$ & $82.7 \dagger$ & $74.2,88.9$ & 0.2 & $0.0,0.9$ & $11 \cdot 1$ & $7 \cdot 3,16 \cdot 6$ & & \\
\hline Medium & 681 & 45.7 & $30 \cdot 3$ & $24.7,36 \cdot 6$ & $47 \cdot 4^{*}$ & $40 \cdot 8,54 \cdot 2$ & $77 \cdot 8^{\star}$ & $71 \cdot 8,82 \cdot 8$ & $2 \cdot 5^{\star}$ & $0.9,6.4$ & $16 \cdot 9$ & $11 \cdot 6,24 \cdot 0$ & & \\
\hline High & 235 & $15 \cdot 8$ & $39 \cdot 3$ & $28.9,50.9$ & $24 \cdot 3$ & $16 \cdot 6,34 \cdot 1$ & 63.7 & $51 \cdot 4,74.4$ & 0.0 & $0.0,0.0$ & 8.0 & $2 \cdot 5,22 \cdot 4$ & & \\
\hline Without information & 237 & $15 \cdot 9$ & $46 \cdot 3$ & $34 \cdot 0,59 \cdot 1$ & $24 \cdot 2$ & $16 \cdot 3,34 \cdot 3$ & 70.5 & $59 \cdot 2,79 \cdot 7$ & 0.0 & $0.0,0.4$ & 5.8 & $2 \cdot 4,13 \cdot 3$ & & \\
\hline \multicolumn{15}{|l|}{ Education level } \\
\hline Low $(<8$ years) & 112 & 7.5 & $27 \cdot 1$ & $16 \cdot 4,41 \cdot 3$ & $65 \cdot 5 \dagger \neq$ & $50 \cdot 6,77 \cdot 9$ & $92 \cdot 6 \dagger \ddagger$ & $82 \cdot 1,97 \cdot 1$ & 0.5 & $0.1,2.9$ & $31.0 \dagger \ddagger$ & $17 \cdot 9,48 \cdot 2$ & $7 \cdot 0$ & $0.0,19 \cdot 6$ \\
\hline Medium (8-12 years) & 832 & $55 \cdot 8$ & $36 \cdot 2$ & $30 \cdot 6,42 \cdot 2$ & $43 \cdot 6^{*}$ & $37.8,49.6$ & $79 \cdot 9^{*}$ & $74.6,84 \cdot 3$ & 1.2 & $0.5,5.0$ & $14 \cdot 7^{\star}$ & $10.4,20.5$ & $6 \cdot 6$ & $1.9,11.3$ \\
\hline High (13 years or more) & 546 & $36 \cdot 7$ & $39 \cdot 3$ & $31 \cdot 8,47 \cdot 4$ & $26 \cdot 0$ & $20 \cdot 1,33 \cdot 0$ & 65.4 & $57 \cdot 7,72 \cdot 4$ & $0 . \overline{7}$ & $0 \cdot 2,2 \cdot 6$ & $5 \cdot 2$ & $2 \cdot 2,11 \cdot 4$ & $9 \cdot 7^{*}$ & $2 \cdot 9,16 \cdot 4$ \\
\hline \multicolumn{15}{|l|}{ Ethnicity } \\
\hline Indigenous & 128 & $8 \cdot 6$ & $25 \cdot 1$ & $17 \cdot 4,34 \cdot 7$ & $55 \cdot 8^{\star \star}$ & $44 \cdot 4,66 \cdot 6$ & 80.9 & $71 \cdot 2,87 \cdot 9$ & 0.0 & $0.0,0.0$ & $10 \cdot 0$ & $5 \cdot 5,17 \cdot 6$ & & \\
\hline Non-indigenous & 1361 & 91.4 & 37.8 & $33 \cdot 1,42 \cdot 7$ & 37.2 & $32 \cdot 7,42 \cdot 0$ & $75 \cdot 0$ & $70.5,79.0$ & 1.3 & $0.5,3.2$ & $12 \cdot 7$ & $9 \cdot 3,16 \cdot 9$ & & \\
\hline \multicolumn{15}{|l|}{ Women $>49$ years } \\
\hline $\begin{array}{l}\text { All } \\
\text { Hous }\end{array}$ & 1768 & $100 \cdot 0$ & $38 \cdot 1$ & $33 \cdot 9,42 \cdot 5$ & $45 \cdot 0$ & $40 \cdot 6,49 \cdot 5$ & $83 \cdot 1$ & $79 \cdot 5,86 \cdot 1$ & 0.7 & $0.3,1.8$ & & & $5 \cdot 1$ & $0.0,10.7$ \\
\hline $\begin{array}{l}\text { Low } \\
\text { Lousenta income }\end{array}$ & 690 & 39.0 & $32.0 \ddagger$ & $27 \cdot 1,37 \cdot 3$ & 49.5 & $43 \cdot 5,55 \cdot 5$ & 81.5 & $75 \cdot 3,86 \cdot 4$ & 0.5 & $0.2,1.5$ & & & & \\
\hline Medium & 672 & 38.2 & $45 \cdot 1$ & $37 \cdot 3,53 \cdot 1$ & 41.7 & $34.0,49.7$ & $86 \cdot 8^{*}$ & $81 \cdot 7,90 \cdot 6$ & 1.2 & $0.3,4.6$ & & & & \\
\hline High & 104 & 5.9 & $32 \cdot 1$ & $19 \cdot 3,48 \cdot 2$ & 37.9 & $21 \cdot 9,57 \cdot 1$ & $70 \cdot 0$ & $53 \cdot 1,82 \cdot 8$ & $0 . \overline{0}$ & $0.0,0.0$ & & & & \\
\hline Without information & 302 & $17 \cdot 1$ & 38.3 & $27 \cdot 5,50 \cdot 5$ & 44.7 & $33.4,56.5$ & 83.0 & $72 \cdot 2,90 \cdot 2$ & 0.3 & $0.0,1.8$ & & & & \\
\hline \multicolumn{15}{|l|}{ Education level } \\
\hline Low ( $<8$ years) & 684 & $38 \cdot 7$ & $31 \cdot 2 † \ddagger$ & $25 \cdot 9,37 \cdot 1$ & $52 \cdot 2 \dagger \ddagger$ & $46 \cdot 0,58 \cdot 3$ & 83.4 & $77 \cdot 9,87 \cdot 8$ & 1.4 & $0.4,4.4$ & & & 5.9 & $0.0,18 \cdot 3$ \\
\hline Medium (8-12 years) & 874 & $49 \cdot 4$ & 41.5 & $34.9,48 \cdot 4$ & 42.1 & $35 \cdot 4,49 \cdot 1$ & 83.6 & $78.1,87.9$ & 0.3 & $0.0,1.2$ & & & $2 \cdot 8$ & $0.0,11.0$ \\
\hline High ( 13 years or more) & 210 & 11.9 & $46 \cdot 0$ & $33 \cdot 7,58 \cdot 8$ & 33.7 & $22 \cdot 5,47 \cdot 1$ & 79.7 & $68 \cdot 3,87 \cdot 8$ & 0.0 & $0.0,0.6$ & & & 0.0 & $0.0,0.0$ \\
\hline \multicolumn{15}{|l|}{ Indigenous origin } \\
\hline Indigenous & 110 & $6 \cdot 2$ & 34.6 & $22 \cdot 9,48 \cdot 4$ & 52.5 & $39.8,64.9$ & $87 \cdot 1$ & $76 \cdot 7,93 \cdot 2$ & 0.0 & $0.0,0.0$ & & & & \\
\hline \multirow{2}{*}{\multicolumn{15}{|c|}{ Men $20-49$ years }} \\
\hline & & & & & & & & & & & & & & \\
\hline All & 842 & $100 \cdot 0$ & 43.5 & $38 \cdot 1,49 \cdot 1$ & $30 \cdot 5$ & $25 \cdot 8,35 \cdot 7$ & $74 \cdot 0$ & $69 \cdot 1,78 \cdot 4$ & 0.9 & $0.3,2 \cdot 2$ & $9 \cdot 4$ & $6 \cdot 7,12 \cdot 9$ & & \\
\hline \multicolumn{15}{|l|}{ Household income } \\
\hline Low & 128 & $15 \cdot 2$ & $37 \cdot 2$ & $26 \cdot 4,49 \cdot 5$ & $35 \cdot 6$ & $24 \cdot 7,48 \cdot 2$ & $72 \cdot 8$ & $62 \cdot 1,81 \cdot 4$ & 3.5 & $1.1,10.5$ & 19.5 & $11 \cdot 3,31 \cdot 7$ & & \\
\hline Medium & 357 & $42 \cdot 4$ & 43.4 & $35 \cdot 6,51.6$ & 30.6 & $24 \cdot 0,38 \cdot 1$ & 74.0 & $66 \cdot 7,80 \cdot 2$ & 0.0 & $0.0,0.0$ & $8.6+$ & $5 \cdot 1,14 \cdot 3$ & & \\
\hline High & 226 & 26.9 & 43.4 & $32 \cdot 2,55 \cdot 4$ & 32.5 & $22.4,44.5$ & 75.9 & $64.6,84.5$ & 0.0 & $0.0,0.0$ & 6.8 & $2 \cdot 8,15 \cdot 3$ & & \\
\hline Without information & 131 & $15 \cdot 6$ & $50 \cdot 0$ & $36 \cdot 2,64 \cdot 0$ & 21.9 & $13 \cdot 2,33.9$ & $72 \cdot 0$ & $57 \cdot 3,83 \cdot 1$ & $2 \cdot 3$ & $0.5,10 \cdot 2$ & $6 \cdot 0$ & $2.5,13.9$ & & \\
\hline
\end{tabular}


Table 2 Continued

\begin{tabular}{|c|c|c|c|c|c|c|c|c|c|c|c|c|c|c|}
\hline & \multirow{2}{*}{\multicolumn{2}{|c|}{ All }} & \multicolumn{6}{|c|}{ Excess of weight } & \multicolumn{6}{|c|}{ Undernutrition } \\
\hline & & & \multicolumn{2}{|c|}{ Overweightll } & \multicolumn{2}{|c|}{ Obesity } & \multicolumn{2}{|c|}{ Excess weight†† } & \multicolumn{2}{|c|}{ Underweightł‡ } & \multicolumn{2}{|c|}{ Short stature§§ } & \multicolumn{2}{|c|}{ AnaemiallII } \\
\hline & $n$ & $\% \S$ & $\%$ & $95 \% \mathrm{Cl}$ & $\%$ & $95 \% \mathrm{Cl}$ & $\%$ & $95 \% \mathrm{Cl}$ & $\%$ & $95 \% \mathrm{Cl}$ & $\%$ & $95 \% \mathrm{Cl}$ & $\%$ & $95 \% \mathrm{Cl}$ \\
\hline \multicolumn{15}{|l|}{ Education level } \\
\hline Low ( $<8$ years) & 41 & 4.9 & $18 \cdot 6 \neq \dagger$ & $7 \cdot 7,38.5$ & 35.5 & $15 \cdot 9,61 \cdot 6$ & $54 \cdot 1$ & $29 \cdot 6,76 \cdot 8$ & 4.4 & $0 \cdot 6,25 \cdot 8$ & $47 \cdot 6 \neq \dagger$ & $24 \cdot 6,71 \cdot 6$ & & \\
\hline Medium (8-12 years) & 441 & $52 \cdot 4$ & $45 \cdot 5$ & $38 \cdot 5,52 \cdot 7$ & $32 \cdot 2$ & $26 \cdot 1,38 \cdot 9$ & $77 \cdot 7$ & $71 \cdot 7,82 \cdot 7$ & 1.2 & $0.4,3.6$ & $9 \cdot 8^{\star}$ & $6 \cdot 5,14.6$ & & \\
\hline High ( 13 years or more) & 359 & $42 \cdot 7$ & $44 \cdot 0$ & $35 \cdot 3,53 \cdot 0$ & $27 \cdot 9$ & $20 \cdot 5,36 \cdot 7$ & 71.8 & $63 \cdot 3,79 \cdot 1$ & 0.0 & $0.0,0.5$ & 4.5 & $2 \cdot 1,9.5$ & & \\
\hline \multicolumn{15}{|l|}{ Ethnicity } \\
\hline Indigenous & 77 & $9 \cdot 1$ & $36 \cdot 9$ & $24 \cdot 6,51 \cdot 3$ & $42 \cdot 4$ & $29 \cdot 4,56 \cdot 6$ & $79 \cdot 3$ & $66 \cdot 4,88 \cdot 2$ & $0 \cdot 0^{* *}$ & $0.0,0.0$ & $21 \cdot 5^{\star \star}$ & $11 \cdot 9,35 \cdot 7$ & & \\
\hline Non-indigenous & 765 & $90 \cdot 9$ & $44 \cdot 2$ & $38.4,50 \cdot 1$ & $29 \cdot 3$ & $24 \cdot 3,34.8$ & 73.5 & $68 \cdot 2,78 \cdot 2$ & 1.0 & $0.4,2.5$ & 8.2 & $5.5,11.9$ & & \\
\hline \multicolumn{15}{|l|}{ Men $>49$ years } \\
\hline All & 983 & $100 \cdot 0$ & $48 \cdot 3$ & $42 \cdot 7,54 \cdot 0$ & 33.7 & $28 \cdot 5,39 \cdot 3$ & $82 \cdot 0$ & $77 \cdot 6,85 \cdot 7$ & 0.3 & $0.1,1.0$ & & & & \\
\hline \multicolumn{15}{|l|}{ Household income } \\
\hline Low & 236 & $24 \cdot 0$ & 44.1 & $36 \cdot 0,52 \cdot 5$ & $32 \cdot 2$ & $24 \cdot 6,40 \cdot 8$ & $76 \cdot 3$ & $69 \cdot 0,82 \cdot 3$ & 0.4 & $0.0,2 \cdot 3$ & & & & \\
\hline Medium & 475 & $48 \cdot 3$ & $49 \cdot 5$ & $41 \cdot 0,58 \cdot 0$ & $32 \cdot 0$ & $24 \cdot 6,40 \cdot 3$ & 81.5 & $74 \cdot 1,87 \cdot 1$ & 0.5 & $0.0,1.9$ & & & & \\
\hline High & 137 & $13 \cdot 9$ & $47 \cdot 3$ & $29.6,65 \cdot 7$ & 37.5 & $21 \cdot 3,57 \cdot 2$ & 84.9 & $67 \cdot 4,93 \cdot 8$ & 0.0 & $0.0,0.0$ & & & & \\
\hline Without information & 135 & $13 \cdot 8$ & 52.4 & $38 \cdot 0,66 \cdot 4$ & 38.4 & $25 \cdot 3,53 \cdot 4$ & $90 \cdot 8$ & $81 \cdot 1,95 \cdot 8$ & 0.0 & $0.0,0.0$ & & & & \\
\hline \multirow{2}{*}{\multicolumn{15}{|c|}{ Indigenous origin }} \\
\hline & & & & & & & & & & & & & & \\
\hline Low ( $<8$ years) & 302 & $30 \cdot 8$ & 44.1 & $35 \cdot 2,53 \cdot 4$ & 35.5 & $27 \cdot 2,44.9$ & 79.7 & $71 \cdot 7 ; 85 \cdot 8$ & 0.4 & $0.1,1.7$ & & & & \\
\hline Medium (8-12 years) & 471 & 47.9 & $51 \cdot 2$ & $43 \cdot 3,59 \cdot 1$ & $29 \cdot 6$ & $22 \cdot 8,37 \cdot 3$ & $80 \cdot 8$ & $74 \cdot 1,86 \cdot 1$ & 0.4 & $0 \cdot 1,2 \cdot 0$ & & & & \\
\hline High ( 13 years or more) & 210 & $21 \cdot 3$ & $47 \cdot 8$ & $33 \cdot 9,62 \cdot 1$ & $40 \cdot 3$ & $27 \cdot 2,55 \cdot 0$ & $88 \cdot 1$ & $76 \cdot 3,94 \cdot 4$ & 0.0 & $0.0,0.0$ & & & & \\
\hline \multicolumn{15}{|l|}{ Ethnicity } \\
\hline Indigenous & 77 & 5.6 & $46 \cdot 1$ & $23 \cdot 3,70 \cdot 7$ & 34.9 & $16 \cdot 8,58 \cdot 6$ & $81 \cdot 0$ & $63.0,91.5$ & 1.5 & $0.2,10 \cdot 6$ & & & & \\
\hline Non-indigenous & 928 & 94.4 & $48 \cdot 4$ & $42 \cdot 7,54 \cdot 2$ & 33.6 & $28 \cdot 3,39 \cdot 4$ & $82 \cdot 0$ & $77 \cdot 4,85 \cdot 9$ & 0.2 & $0.0,1.0$ & & & & \\
\hline
\end{tabular}

§Weighted \%.

|lOverweight: BMI $\geq 25$ and $<30 \mathrm{~kg} / \mathrm{m}^{2}$ for women and men.

ПObesity: BMI $\geq 30 \mathrm{~kg} / \mathrm{m}^{2}$ for women and men.

TObesity: BMl $\geq 30 \mathrm{~kg} / \mathrm{m}^{2}$ for women and men.
t+Overweight/obesity: $\mathrm{BMI} \geq 25 \mathrm{~kg} / \mathrm{m}^{2}$ for women and men.
t+Underweight: $\mathrm{BMl}<18.5 \mathrm{~kg} / \mathrm{m}^{2}$ for women and men.

\&\&Short stature: height $<1.49 \mathrm{~m}$ for women $20-49$ years, height $<1.62 \mathrm{~m}$ for men $20-49$ years.

IIIIAnaemia: defined as $<120 \mathrm{~g} / \mathrm{l}$ for women. The sample size for anaemia was 829 for women $20-49$ years and 910 for women +50 years. The data were based on the 2003 National Health Survey (ENS).

${ }^{*} P$ value $<0.05$ medium $v$. high.

$\dagger P<0.05$ high $v$. low.

${ }_{* *}^{*}$ Indigenous $v$. non-indigenous. 
Malnutrition inequalities in Chilean adults

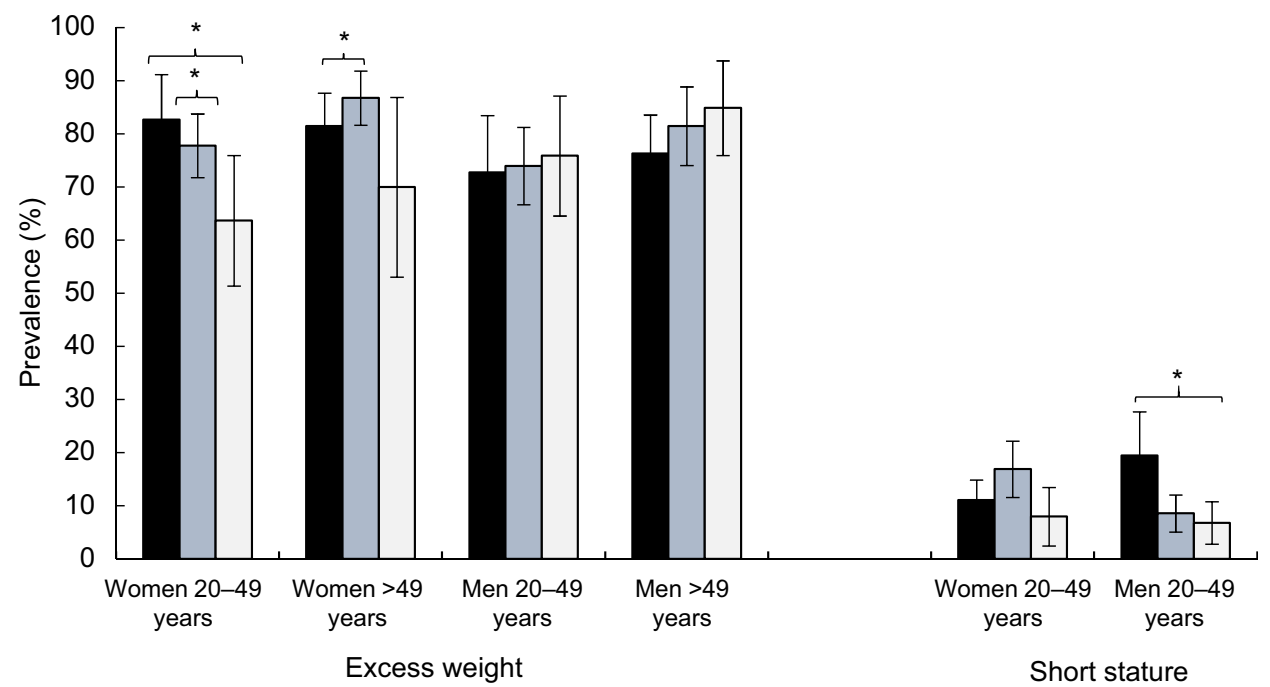

Fig. 1 (colour online) Prevalence of excess weight and short stature in men and women by income. Low, low income level, medium;

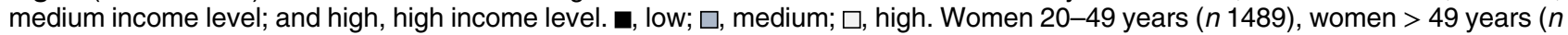
1768), men $20-49$ years ( $n$ 842), men $>49$ years $\left(n\right.$ 983). ${ }^{*} P$ value $<0.05$

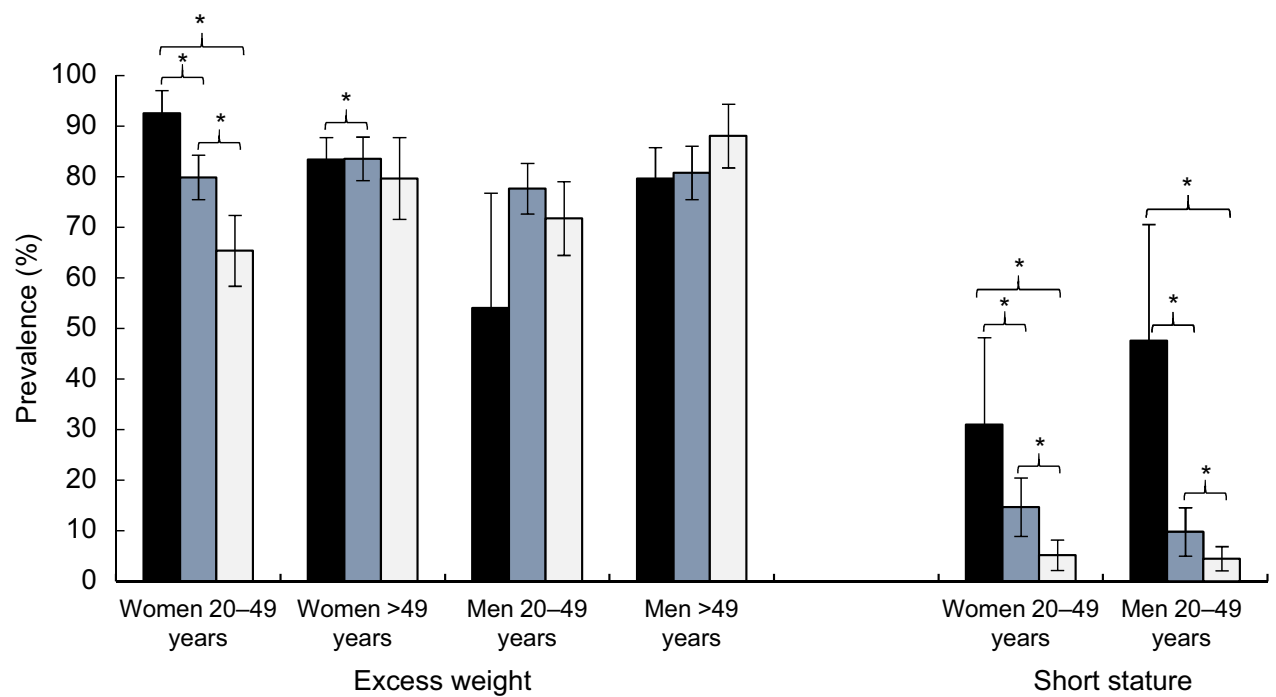

Fig. 2 (colour online) Prevalence of excess weight and short stature in men and women by education level. $\mathbf{\square}$, low (0-7 years); $\square$, medium (8-12 years); $\square$, high (>12 years). Women 20-49 years ( $n$ 1489), women $>49$ years $(n 1768)$, men 20-49 years ( $n$ 842), men $>49$ years $(n 983)$. ${ }^{*} P$ value $<0.05$

$P<0.05)$; however, these differences did not reach statistical differences among older women $(P>0 \cdot 05)$ (Fig. 3).

In young women $(<49$ years $)$, short stature was more frequent among low-educated women $(P<0 \cdot 05)$, while undernutrition was more frequent among mid-educated women $(P<0.05)$ and anaemia among high-educated women $(P<0 \cdot 05)$. No significant differences in undernutrition indicators were observed in older women ( $>49$ years; $P>0.05$ ) by SES (Table 2).

\section{Nutrition status, socioeconomic status and etbnicity in men}

In men, excess weight did not significantly differ by SES or ethnicity, except for a lower prevalence of overweight among the young men ( $<49$ years) with low education $(P<0.05$, Figs 1 and 2$)$.

In the case of undernutrition indicators, we observed an inverse relationship between education and short stature $(P<0.05)$; short stature was also more prevalent among indigenous men than in non-indigenous $(P<0.05)$ (Fig. 3).

\section{Discussion}

Our results showed that Chile presents a high prevalence of excess weight (78.7\% in women and $77.2 \%$ in men), as well as a low prevalence of undernutrition (anaemia in women $(7 \cdot 2 \%)$, short stature (13\%) and underweight $(<1 \%)$ in men and women). Compared with other 


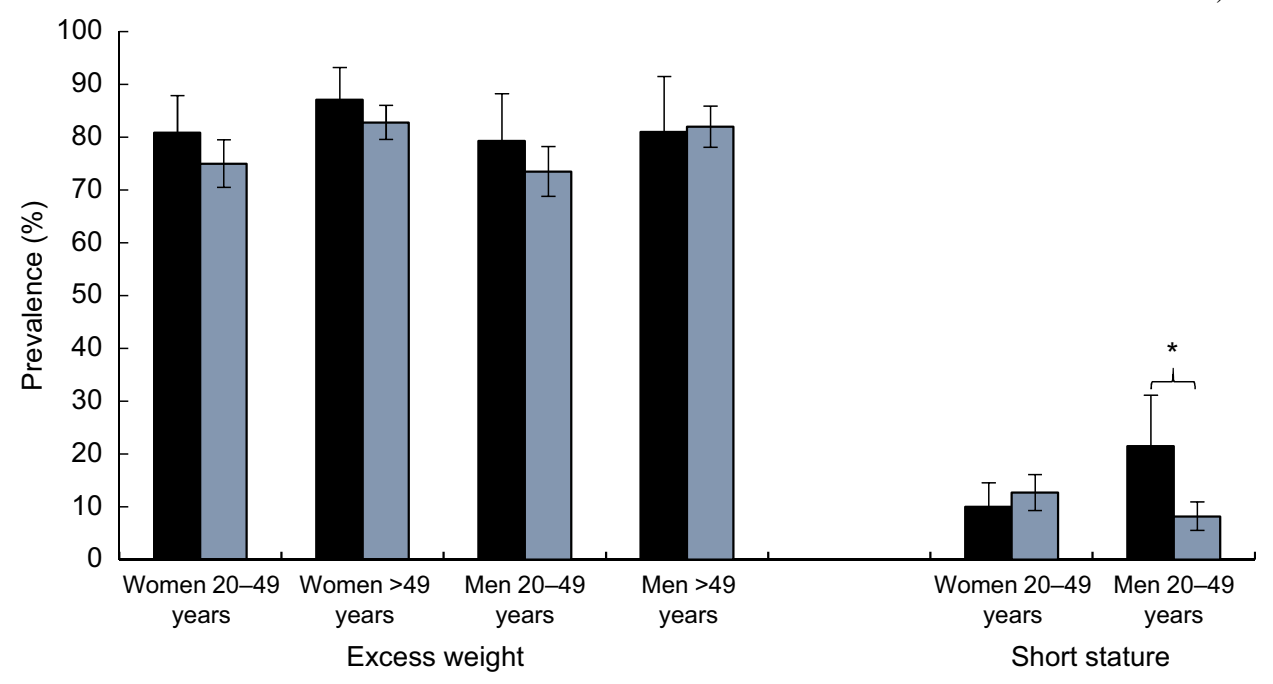

Fig. 3 (colour online) Prevalence of excess weight and short stature in men and women by ethnicity. $\mathbf{\square}$, indigenous; $\square$, nonindigenous. Women 20-49 years ( $n$ 1489), women $>49$ years $(n 1768)$, men 20-49 years $(n 842)$, men $>49$ years $(n$ 983). ${ }^{\star} P$ value $<0.05$

countries in the region, the prevalence of obesity and excess weight in the Chilean population is critically high. Our results are only comparable with those reported in Mexican and Salvadorian adult population, in which 71 and $33 \%$ of women aged $>20$ years had excess weight and obesity, respectively ${ }^{(36)}$, while other Latin American countries have shown a lower prevalence of obesity and excess weight in adults. In contrast, the prevalence of anaemia in childbearing age women and underweight is the lowest in the region ${ }^{(37)}$. Additionally, compared with other countries in the region, for example, Mexico, where the prevalence of short stature is significantly high in low education and low SES adults compared with those with high SES $^{(38)}$, Chilean population statures seems to be linked with socio-economic factors, showing that people from lower SES are more likely to be shorter that people from higher SES. As a result, the double burden situation does not exist in Chile anymore ${ }^{(11)}$; similar trends of the nutrition situation have been described in post-transitional countries in which undernutrition problems are eradicated but replaced by excess weight issues.

Potential explanations for this situation are related to the rapid nutritional transition experienced by Chilean population $^{(4,5)}$. In 1960s, the prevalence of undernutrition in children (<6 years) was $37 \%$, dropping to $2.9 \%$ in $2000^{(2)}$. Successful policies and programs such as the universal access to the healthcare system and the implementation of national complementary food programs have been key strategies to eradicate undernutrition in the country ${ }^{(39)}$. The improvement of sanitary conditions (e.g. sewage system $)^{(40,41)}$ and the mandatory Fe wheat flour fortification (30 mg of $\mathrm{Fe} / \mathrm{kg}$ of wheat flour) have been described as key public health actions to prevent anaemia and underweight in vulnerable population ${ }^{(42,43)}$. Despite these successful results, Chile is facing an alarming prevalence of excess weight ${ }^{(7)}$. It is well-known that several factors influence the nutritional status (e.g. dietary intake, socioeconomic status, healthcare facilities) ${ }^{(44)}$. Over the last decades, Chile has experienced rapid economic changes. Between 1980 and 2014, the average rate of economic growth was $5 \%$, being the highest rate in Latin America ${ }^{(18)}$. This situation has influenced the population's dietary patterns, increasing the consumption of energy-dense, sugar and fat-rich food, known as a 'Western diet'(45). Trade liberalisation and rapid expansion of supermarkets and fast-food restaurants have profoundly influenced the dietary pattern ${ }^{(46)}$. Also, the increase in income in families has promoted greater portion sizes and away-home food intake ${ }^{(8,47)}$. Concomitant with the changes of food habits, the prevalence of sedentary lifestyle had continuously increase affecting more than $80 \%$ of adult Chilean population ${ }^{(7)}$.

We found a higher prevalence of obesity and excess weight in women compared with men, while men population had a higher prevalence of overweight than women. Previous research has indicated a complex relationship between sex, SES and the prevalence of excess weight and obesity ${ }^{(48)}$. On one side, it has been shown that women are more likely to choose added sugar and high-fat content foods compared with men, which may promote the weight gain due to an excess of energy intake ${ }^{(22)}$. Additionally, men classified in the low socioeconomic level had shown higher rates of occupation compared with women. Occupation in the lowest socioeconomic level population remained a significant source of physical activity and subsequently a higher energy expenditure, which may explain the differences found in the prevalence of obesity compared by sex in the low SES group ${ }^{(22)}$.

However, not only dietary intake and physical activity may explain these differences, cultural factors, such as the role of woman in the society and differences on bodyweight concerns, self-esteem ${ }^{(49)}$ and dissatisfaction of their 
body by sex ${ }^{(50)}$ also define particular views of the beauty that do not necessarily follow biomedical standards may also contribute to explain our findings ${ }^{(51)}$. An interesting work recently published by Keller et al. ${ }^{(52)}$ in children showed that encouragement to eat is reinforced in males but not in females. Additionally, their findings indicated that females have a higher neural reactivity to high-energy food compared with male children, which may contribute to the higher risk of obesity in females than males later in life.

In addition, our results showed that poorer and less educated women had a higher prevalence of obesity compared with the richest, while in men, no significant differences were found in obesity and excess weight prevalence's comparing by income and educational level. Contrary, less educated men had a lower prevalence of overweight compared with those with a higher educational level. Differences in malnutrition prevalence by sex and SES have been also shown in other countries of the region. Results of a novel modelling of nutrition stages dynamics in Colombian population showed that the burden of excess weight is likely higher in those with lower SES ${ }^{(53)}$. Additionally, Hernandez et $a l .{ }^{(54)}$ found that food insecure women had a greater percentage of obesity and overweight compared with food secure women, while among men the proportion of obesity and overweight was similar between food secure and food insecure participants.

Female sex is a well-known risk factor for inequity and poverty ${ }^{(55)}$. Findings from a large study conducted by Wells et al. ${ }^{(56)}$ which included data from sixty-eight countries showed that wealth and sex inequalities are associated with the prevalence of obesity in women. Despite the rapid economic growth, Chile is affected by a dramatic income inequality. It has been reported that the income distribution is fourteen times lower in the poorer quartile compared with the richest ${ }^{(17)}$. In the USA, it has been described that poorer people have a less varied diet and less access to healthier food ${ }^{(57)}$. Low cost and easily accessible food are characterised by a high content of sugar, low-quality fat and increased palatability, which may explain a higher energy intake and a subsequent higher prevalence of obesity in poorer adults. However, women have a higher risk of food insecurity and inadequate dietary intake which may promote buying low cost and high-energy dense food. Also, it has been described that low SES woman has reduced participation in food's budget leading to poorer nutrient-quality diets ${ }^{(56)}$, which may partially explain the sex differences in the prevalence of obesity in low SES adults.

We also found that richer and higher educated women had a lower prevalence of excess weight and short stature compared with the poorer group, while men with a higher income and education level were less likely to have short stature compared with the poorest and lowest education level group. It has been speculated that women with a higher educational level might develop obesity-preventing health behaviour, as well as better healthcare access ${ }^{(58)}$ that may partially explain the lower prevalence of excess weight. Additionally, a potential explanation for the link between SES and short stature is that height is closely related to a variation in net nutrition, meaning that poorer people probably have a more deficient diet, affecting early development and stature ${ }^{(59,60)}$.

For the first time in Chile, we are showing in a national representative sample of adults that indigenous women (<49 years) have a higher prevalence of obesity, while men ( $<49$ years) have a higher prevalence of short stature compared with non-indigenous population. It has been previously described a complex interaction between ethnicity, inequality and sex differences in indigenous population in Chile. Indigenous tend to have a lower SES and education level, which may affect the variety of the diet and the preference for low-cost and ultra-processed foods which may promote a positive energy balance and a subsequent higher risk of obesity in women. Additionally, a recent study conducted by Fernández et al. ${ }^{(61)}$ showed that indigenous boys have a higher risk of precocious gonadarche and pubarche. In European and US boys ${ }^{(62,63)}$, early puberal onset has been inversely associated with height in adulthood, which may explain the shorter height that we found in indigenous men compared with non-indigenous; higher adversity during infancy and adolescence may also contribute to long-term short stature.

\section{Current programs and policies focused on preventing obesity}

In Chile, there are several initiatives in place to promote breast-feeding, a known protective factor of obesity for both the children and the mother ${ }^{(64-67)}$. Baby Friendly Hospitals $^{(68)}$ that promote early bonding and latching ensure perinatal breast-feeding education, breast-feeding consultancy after birth and control the early introduction of formula, breast-feeding education to pregnant women and breast-feeding support for breast-feeding mothers, including lactating clinics, through primary healthcare and the multi-sectoral program 'Chile Crece Contigo'(68), and a six months paid maternity leave ${ }^{(69)}$. There is also the 'Vida Sana' program that was one of the first strategies that Chile developed against obesity. This program offers free-charge dietitian, medical and physical interventions in public healthcare centers for population between 2 and 64 years. Considering that obesity is a multifactorial disease, in 2006, the Chilean government endeavoured the implementation of a public-private strategy, 'Estrategia Global contra la Obesidad' (EGO), as a multi-sectorial and multidisciplinary collaborative initiative between the Chilean Ministry of Health and the WHO. This strategy was aimed to tackle the high rates of obesity and chronic diseases through the promotion of healthy diet and active lifestyle. Additionally, as an effort to promote healthy eating patterns and ensure healthier environments for children, 
Chile implemented the first food labelling and advertising law (Law 20.606), which included the use of a warning label on packaged foods with excess calories, sodium, sugars or saturated fats as well as restricted marketing of those foods to children and restriction to sell or promote those foods in nursery schools or schools ${ }^{(8,70,71)}$.

All these strategies have faced several challenges for an adequate implementation and do not consider evaluation components as part of their implementation. There have been several efforts to evaluate these policies, but evaluation components need to be incorporated in the phase of the design to be able to properly assess the effectiveness of the different actions, considering a sex, SES and ethnicity approach. This information is key to prevent inefficient allocation of valuable resources and to improve the current public health actions. Our study should contribute in highlighting high-risk groups in which to focus action as we included a large and national-representative data of women and men population. Our study also highlights the need of collecting updated data on anaemia prevalence in women of childbearing age as well as extending data collection on children to better monitor the nutritional trends in the country.

\section{Conclusions}

Currently, Chilean adult population presents a high prevalence of obesity and overweight while undernutrition (including anaemia) is low. Nutrition status of the population varies importantly by sex, SES and ethnicity highlighting the need that future policies and programs address these important inequalities.

\section{Acknowledgements}

Acknowledgements: The authors thank the Ministry of Health of Chile for supplying the databases (Chilean National Health Surveys 2010 and 2003). Also, the authors thank the Latin American Nutrition Leadership Program (Programa LILANUT) for the coordination of this supplement. We declare not receiving sponsorship from any industry. Financial support: The current study is a secondary analysis of databases, and no specific funds were available for this purpose. Conflicts of interest: M.F.M.-C., D.N.-R., S.L.-A. and C.C. have no conflicts of interest. Authorship: C.C. and M.F.M.-C. designed the research; D.N-R. and S.L.-A. led the data compilation; S.L.-A. led the statistical analyses; C.C., D.N-R. and S.L.-A. provided input on study execution; M.F.M.-C. wrote the first draft of the manuscript; C.C., M.F.M.-C., D.N-R. and S.L.-A. led the interpretation of the data and manuscript completion. All authors contributed to data interpretation and manuscript writing and read and approved the final manuscript. Ethics of buman subject participation: The National Health
Surveys and their informed consents were approved by the Ethics Committee of the Medicine School of the Faculty of Medicine, Pontifical Catholic University of Chile before its execution, in accordance with the Helsinki Declaration. The National Health Surveys data are available by the Epidemiology Department, Ministry of Health with no identifiable information on survey's participants.

\section{References}

1. Kaempffer A \& Medina E (2006) Recent infant mortality rate in Chile: successes and challenges. Rev Chil Pediatr 77, 492-500.

2. Willett WC (1997) Public health benefits of preventive nutrition. In Preventive Nutrition, 4th ed., pp. 3-22 [A Bendich \& RJ Deckelbaum, editors]. New York: Humana Press.

3. Ríos E, Olivares M, Amar M et al. (1983) Evaluation of iron status and prevalence of iron deficiency in infants in Chile. In Nutrition Interventions Strategies in National Development, 1st ed., pp. 273-283 [BA Underwood, editor]. New York: Academic Press.

4. Albala C, Vio F, Kain J et al. (2001) Nutrition transition in Latin America: the case of Chile. Nutr Rev 59, 170-176.

5. Vio F, Albala C \& Kain J (2008) Nutrition transition in Chile revisited: mid-term evaluation of obesity goals for the period 2000-2010. Public Health Nutr 11, 405-412.

6. GBD 2015 Obesity Collaborators, Afshin A, Forouzanfar MH et al. (2017) Health effects of overweight and obesity in 195 countries over 25 years. $N$ Engl J Med 377, 13-27.

7. Chilean Ministry of Health. 2016-2017 II National Health Survey. https://www.minsal.cl/portal/ (accessed June 2019).

8. Corvalán C, Garmendia ML, Jones-Smith J et al. (2017) Nutrition status of children in Latin America. Obes Rev 18, Suppl. 2, 7-18.

9. Chilean National School Board Program (JUNAEB) (2019). Chilean Ministry of Education.

10. Chilean Ministry of Health. 2003 I National Health Survey. https://www.minsal.cl/portal/ (accessed June 2019).

11. Atalah E, Amigo H \& Bustos P (2014) Does Chile's nutritional situation constitute a double burden? Am J Clin Nutr 100(6), 1623S-1627S.

12. Garmendia ML, Alonso FT, Kain J et al. (2014) Alarming weight gain in women of a post-transitional country. Public Health Nutr 17, 667-673.

13. Lanas F, Bazzano L, Rubinstein A et al. (2016) Prevalence, distributions and determinants of obesity and central obesity in the Southern Cone of America. PLoS One 11, $1-12$.

14. Freire WB, Silva-Jaramillo KM, Ram J et al. (2014) The double burden of undernutrition and excess body weight in. Am J Clin Nutr 100, 1636-1643.

15. Ramirez-Zea M, Kroker-Lobos MF, Close-Fernandez R et al. (2014) The double burden of malnutrition in indigenous and nonindigenous. Am J Clin Nutr 100, 1644-1651.

16. Severi C \& Moratorio X (2014) Double burden of undernutrition and obesity in Uruguay. Am J Clin Nutr 100(6), 1659S$1662 S$.

17. Gammage S, Alburquerque T \& Durán G (2014) Poverty, inequality and employment in Chile. International Labour Office (ILO). Conditions of Work and Employment Series, Geneva no. 46, ISSN: 2226-8944; 2226-8952.

18. De Gregorio J (2005) Economic growth in Chile: evidence, sources and perspectives. Estud públicos 98, 19-86.

19. The Organisation for Economic Co-operation and Development (OECD) (2015) In it together. Why less inequality benefits all. Overview of Inequality Trends, Key Findings and Policy directions. 
20. Amarante V (2016) Income inequality in Latin America: a factor component analysis. Rev Income Wealth $\mathbf{6 2}$, Suppl. 1, S4-S21.

21. Wang Y \& Beydoun MA (2007) The obesity epidemic in the United States - gender, age, socioeconomic, racial/ethnic, and geographic characteristics: a systematic review and meta-regression analysis. Epidemiol Rev 29, 6-28.

22. Kanter R \& Caballero B (2012) Global gender disparities in obesity: a review. Adv Nutr 3, 491-498.

23. Aitsi-Selmi A, Batty GD, Barbieri MA et al. (2013) Childhood socioeconomic position, adult socioeconomic position and social mobility in relation to markers of adiposity in early adulthood - evidence of differential effects by gender in the 1978/79 Ribeirao Preto cohort study. Int J Obes 37, 439-447.

24. Barros AJD, Victora CG, Horta BL et al. (2006) Effects of socioeconomic change from birth to early adulthood on height and overweight. Int J Epidemiol 35, 1233-1238.

25. Bustos P, Munoz S, Vargas C et al. (2009) Poverty and indigenous origin as risk factors of nutritional problems among children who enroll in school. Salud Publica Mex 51, 187-193.

26. Azar A, Franetovic G \& Santos H (2015) Individual, social and environmental determinants of overweight and obesity among Chilean adolescents. Rev Méd Chile 146, 598-605.

27. Amigo H, Vásquez A \& Bustos P (2018) Changes in socio-economic level and their impact on nutritional status: a follow-up study among young Chilean adults. Public Health Nutr 21, 2402-2408.

28. The Chilean National Institute of Statistics (INE) (2017) Census.

29. Kain J, Leyton B, Baur L et al. (2019) Demographic, social and health-related variables that predict normal-weight preschool children having overweight or obesity when entering primary education in Chile. Nutrients 11, 1277.

30. Amigo H, Erazo M \& Bustos P (2000) Stature of Chilean parents and children of different ethnicity and social vulnerability. Salud Publica Mex 42, 504-510.

31. World Health Organization. Obesity and Overweight. http:// www.who.int/mediacentre/factsheets/fs311/en/ (accessed January 2019).

32. Sorkin JD, Muller DC \& Andres R (1999) Longitudinal change in height of men and women: implications for interpretation of the body mass index: the baltimore longitudinal study of aging. Am J Epidemiol 150, 969-977.

33. Pan American Health Organisation (2011) Underweight, short stature, and overweight in adolescents and young women in Latin America and the Caribbean. 12.

34. Blümel JE, Chedraui $\mathrm{P}$, Calle A et al. Age at menopause in Latin America. Menopause 13, 706-712.

35. Chan M (2011) Haemoglobin Concentrations for the Diagnosis of Anaemia and Assessment of Severity. Geneva, Switzerland: World Health Organization.

36. Ng M, Fleming T, Robinson M et al. (2014) Global, regional, and national prevalence of overweight and obesity in children and adults during 1980-2013: a systematic analysis for the Global Burden of Disease Study 2013. Lancet 384, 766-781.

37. Mujica-Coopman MF, Brito A, López de Romaña D et al. (2015) Prevalence of anemia in Latin America and the Caribbean. Food Nutr Bull 36, Suppl. 2, S119-S128.

38. Castro-Porras LV, Rojas-Russell ME, Aedo-Santos Á et al. (2018) Stature in adults as an indicator of socioeconomic inequalities in Mexico. Rev Panam Salud Publica/Pan Am J Public Heal 42, 1-9.

39. Brito A, Olivares M, Pizarro T et al. (2013) Chilean complementary feeding program reduces anemia and improves iron status in children aged 11 to 18 months. Food Nutr Bull $\mathbf{3 4}$ 378-385.
40. Schlesinger L, Weinberger J, Figueroa G et al. (1983) Environmental sanitation: a nutrition intervention. In Nutrition Intervention Strategies in National Development, pp. 241 [BA Underwood, editor]. New York: Academic Press, Inc.

41. Dangour A, Watson L, Cumming O, et al. (2013) Interventions to improve water quality and supply, sanitation and hygiene practices, and their effects on the nutritional status of children. Cochrane Database Syst Rev 8, Art. No.: CD009382. doi: 10.1002/14651858.CD009382.pub2.

42. Olivares M, Pizarro F, Hertrampf E et al. (2000) Iron food fortification in Chile. Rev Chil Nutr 27, 340-344.

43. Ríos-Castillo I, Brito A, Olivares M et al. (2013) Low prevalence of iron deficiency anemia between 1981 and 2010 in Chilean women of childbearing age. Salud Publica Mex 55, 478-483.

44. Black RE, Victora CG, Walker SP et al. (2013) Maternal and child undernutrition and overweight in low-income and middle-income countries. Lancet 382, 427-451.

45. Popkin BM (2004) The nutrition transition: an overview of world patterns of change. Nutr Rev 62, 140-143.

46. Rebolledo N, Reyes M, Corvalán C et al. (2019) Dietary intake by food source and eating location in low- and middleincome Chilean preschool children and adolescents from Southeast Santiago. Nutrients 11, 1695.

47. Popkin BM \& Reardon T (2018) Obesity and the food system transformation in Latin America. Obes Rev 19, 1028-1064.

48. Garawi F, Devries K, Thorogood N et al. (2014) Global differences between women and men in the prevalence of obesity: Is there an association with gender inequality? Eur J Clin Nutr 68, 1101-1106.

49. Phares V, Steinberg AR \& Thompson JK (2004) Gender differences in peer and parental influences: body image disturbance, self-worth, and psychological functioning in preadolescent children. $J$ Youth Adolesc 33, 421-429.

50. Williamson S \& Delin C (2012) Young children's figural selections: accuracy of reporting and body size dissatisfaction. Int J Eat Disord 29, 80-84.

51. Robinovich J, Ossa X, Baeza B et al. (2018) Embodiment of social roles and thinness as a form of capital: a qualitative approach towards understanding female obesity disparities in Chile. Soc Sci Med 201, 80-86.

52. Keller KL, Kling SMR, Fuchs B et al. (2019) A biopsychosocial model of sex differences in children's eating behaviors. Nutrients 11, 682.

53. Meisel JD, Sarmiento OL, Olaya C et al. (2018) Towards a novel model for studying the nutritional stage dynamics of the Colombian population by age and socioeconomic status. PLoS One 13, 1-22.

54. Hernandez DC, Reesor LM \& Murillo R (2017) Food insecurity and adult overweight/obesity: gender and race/ethnic disparities. Appetite 117, 373-378.

55. Doyal L (2001) Sex, gender, and health: the need for a new approach. Br Med J 323, 1061-1063.

56. Wells JCK, Marphatia AA, Cole TJ et al. (2012) Associations of economic and gender inequality with global obesity prevalence: understanding the female excess. Soc Sci Med 75, 482-490.

57. Larson NI, Story MT, Nelson MC et al. (2009) Disparities in access to healthy foods in the U.S. Am J Prev Med 36, 74-81.e10.

58. Cohen AK, Rai M, Rehkopf DH et al. (2013) Educational attainment and obesity: a systematic review. Obes Rev 14, 989-1005.

59. Perkins JM, Subramanian SV, Smith GD et al. (2016) Adult height, nutrition, and population health. Nutr Rev 74, 149-165.

60. Núñez J \& Pérez G (2015) Trends in physical stature across socioeconomic groups of Chilean boys, 1880-1997. Econ Hum Biol 16, 100-114. 
61. Fernández M, Pereira A, Corvalán C et al. (2019) Precocious pubertal events in Chilean children: ethnic disparities. J Endocrinol Invest 42, 385-395.

62. Silventoinen K, Haukka J, Dunkel L et al. (2008) Genetics of pubertal timing and its associations with relative weight in childhood and adult height: The Swedish Young Male Twins Study. Pediatrics 121, e885-e891.

63. Biro FM, McMahon RP, Striegel-Moore R et al. (2001) Impact of timing of pubertal maturation on growth in black and white female adolescents: The National Heart, Lung, and Blood Institute Growth and Health Study. J Pediatr 138, 636-643.

64. Bel Ka, Wagner C, Feldman $\mathrm{H}$ et al. (2017) Associations of infant feeding with trajectories of body composition and growth. Am J Clin Nutr 106, 491-498.

65. Rzehak P, Oddy W, Mearin M et al. (2017) Infant feeding and growth trajectory patterns in childhood and body composition in young adulthood. Am J Clin Nutr 106, 568-580.

66. Brion MJA, Lawlor DA, Matijasevich A et al. (2011) What are the causal effects of breastfeeding on IQ, obesity and blood pressure? Evidence from comparing high-income with middle-income cohorts. Int J Epidemiol 40, 670-680.

67. Owen C, Martin R, Whincup P et al. (2005) The effect of breast-feeding on mean body mass index throughout life. Am J Clin Nutr 82, 1298-1307.

68. Schellhorn C \& Valdés V (2010) Lactancia materna contenidos técnicos para profesionales de la salud. Capítulo II, Chile.

69. Gobierno de Chile (2011) Ley 20545 Modifica las Normas de Protección a la Maternidad e Incorpora el Permiso Postnatal Parental Chile:gobierno de Chile. https://www.leychile.cl/ Navegar?idNorma=1030936 (accessed January 2019).

70. Corvalán C, Reyes M, Garmendia ML et al. (2013) Structural responses to the obesity and non-communicable diseases epidemic: the Chilean law of food labeling and advertising. Obes Rev 14(S2), 79-87.

71. Corvalán C, Reyes M, Garmendia ML et al. (2018) Structural responses to the obesity and non - communicable diseases epidemic: update on the Chilean law of food labelling and advertising. Obes Rev 20, 367-374. 